\title{
Jejunal humoral immunity in diarrhoea-predominant IBS
}

A study published in Gut has shown that jejunal tissue from patients with diarrhoeapredominant IBS (IBS-D) has higher numbers and activation of B cells and more cells positive for IgG than jejunal tissue from healthy people. In addition, the density of cells positive for IgG antibodies is associated with the number of daily bowel movements.

As little is known about the immune dysfunction involved in IBS, "we designed this study to clarify the participation and role of locally released immunoglobulins," says corresponding author Maria Vicario.

Gene microarray analysis of jejunal biopsy tissue identified 185 genes differentially expressed in patients with IBS-D $(n=13)$ compared with healthy individuals $(n=8)$. Functional interaction analysis highlighted nine pathways that were associated with IBS-D. The second highest scoring pathway related to humoral immune response.

Immunohistochemical analysis of IBS-D jejunal biopsy tissue showed increased numbers of B cells and plasma cells compared with healthy controls.
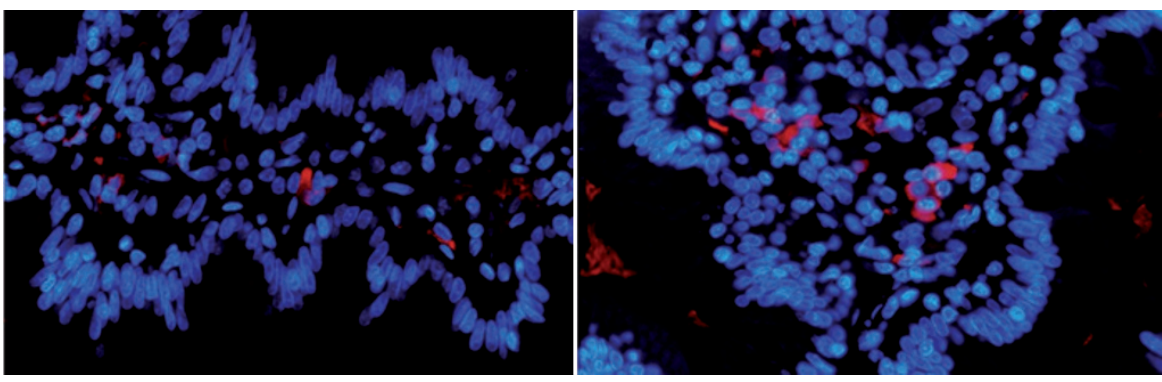

IgG positive cells (red) in healthy (left) and IBS-D jejunum (right). x400 magnification. Image courtesy of M. Vicario.

$\operatorname{IgA}$ and $\operatorname{IgM}$ are the main immunoglobulins produced in the intestinal mucosa. However, the authors found higher mRNA expression of germline transcripts (essential for immunoglobulin heavy chain switching), IgG and IgE in patients with IBS-D than in healthy controls. Immunofluorescent microscopy also showed more cells positive for IgG in the IBS-D group compared with controls.

Interestingly, increases in germline transcripts, IgG mRNA and number of cells expressing IgG antibodies positively correlated with the number of daily bowel movements and stool form in the patients with IBS-D.

Vicario says that future work will aim to identify the immunogens involved in the humoral response. Research investigating new diagnostic tools and therapies for IBS is also planned.

\section{Gillian Patman}

Original article Vicario, M. et al. Increased humoral immunity in the jejunum of diarrhoea-predominant irritable bowel syndrome associated with clinical manifestations. Gut doi:10.1136/gutjnl-2013-306236 